\title{
Complication of vascular access: a case report
}

\section{Sara Milanović Litre*, Adrijana Livaja}

University Hospital Centre Split, Split, Croatia
RECEIVED:

February 19, 2020

ACCEPTED:

February 22, 2020

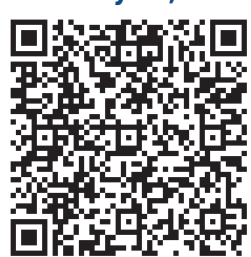

KEYWORDS: vascular access, rupture, complication, stenosis.

CITATION: Cardiol Croat. 2020;15(3-4):67. | https://doi.org/10.15836/ccar2020.67

*ADDRESS FOR CORRESPONDENCE: Sara Milanović Litre, KBC Split, Šoltanska 1, HR-21000 Split, Croatia. / Phone: +385-99-851-36-05 / E-mail: sara.mlitre@gmail.com

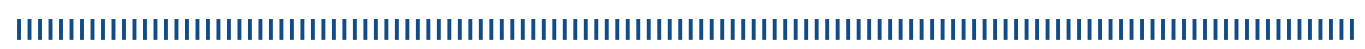

Introduction: Despite technological evolution and intervention techniques, complications resulting from vascular access still occur in patients undergoing percutaneous procedures in invasive laboratories. Brachial artery access, although little used by some specialties, is an option in peripheral vascular procedures and an alternative access in case of failure of other routes. Access site complications lead to longer periods of hospitalization, additional treatments, and higher costs, in addition to being associated with increased morbidity and mortality ${ }^{1}$.

Case report: In this case we have 58-year-old patient with diabetes mellitus and advanced micro and macrovascular complications, history of CABG, and peripheral artery disease was admitted with nonST elevation myocardial infarction complicated with acute heart failure. After initial management a bedside echo was performed that show reduced LVEF of $25 \%$ and hypokinesia of the anterior wall. CT angio was performed which showed patent arterial grafts (LIMA LAD RIMA RCA). Diagnostic coronary angiography was performed using left brachial access which showed significant stenosis of the ostial subclavian artery. Patient was referred to our invasive radiologist, which performed PTA and implanted a stent at the site of stenosis using bi brachial arterial access. Two hours after procedure patient complained about tingling in the fingers of his right hand, with swelling. A CTA was performed and revealed a rupture of the right brachial artery, and a vascular surgeon was called, who sealed the place of bleeding. Patient was discharged 9 days after, and a follow up was performed after one year, with echo showing EFLV of 50\% and patient being angina free.

LITERATURE IIIIIIIIIIIIIIIIIIIIIIIIIIIIIIIIIIIIIIIIIIIIIIIIIIIIIIIIIIIIIIIIIIIIIIIIIIIIIIIIIIIIIIIIIIIIIIIIIIIIII

1. Fonseca P, Almeida J, Bettencourt N, Ferreira N, Carvalho M, Ferreira W, et al. Incidence and predictors of vascular access site complications following transfemoral transcatheter aortic valve implantation. Rev Port Cardiol. 2017 0ct;36(10):747-753. https://doi.org/10.1016/j.repc.2015.11.009 